\title{
Frequency and Risk Factors for Cerebral Arterial Disease in a HIV/AIDS Neuroimaging Cohort
}

\author{
Nancy J. Edwards ${ }^{a}$ Marie F. Grill ${ }^{b}$ H. Alex Choi ${ }^{a}$ Nerissa U. Ko ${ }^{c}$ \\ ${ }^{a}$ Departments of Neurology and Neurosurgery, University of Texas Health Science at Houston, Houston, Tex., \\ ${ }^{b}$ Department of Neurology, Mayo Clinic, Scottsdale, Ariz., and 'Department of Neurology, University of California, \\ San Francisco, Calif., USA
}

\section{Key Words}

Cerebral aneurysms · Cerebrovascular disease · Carotid artery disease $\cdot$ Human immunodeficiency virus and stroke · Vascular imaging $\cdot$ Vasculitis

\begin{abstract}
Background: Infection with HIV predisposes patients to a myriad of neurologic disorders, including cerebrovascular disease. The pathophysiology is likely multifactorial, with proposed mechanisms including infectious vasculitis, HIVinduced endothelial dysfunction and adverse effects of combination antiretroviral therapy (cART). Epidemiologic data on clinically evident cerebral vasculopathy in HIV-infected adults is scarce, even though stroke hospitalizations are rising in this patient population. Methods: A total of 6,298 HIVinfected adults (San Francisco General Hospital, 2000-2013) were screened to generate a cohort of patients with dedicated neuroimaging of the intra- and extracranial cerebral vasculature. We extracted information regarding the extent of HIV disease (including serial viral load and CD4 counts), cardiovascular disease risk factors and exposure to cART (cross-referenced with pharmacy records) and performed multivariate logistic regression analysis to identify predictors of vasculopathy. Results: Of 144 patients, 55 patients
\end{abstract}

(38.2\%) had radiographic evidence of cerebral vasculopathy. Twenty (13.9\%) had a vasculopathy characterized by vessel dolichoectasia and intracranial aneurysm formation. Thirtyfive patients (24.3\%) had intra- and or extracranial stenosis/ occlusion. cART use (OR 2.27, 95\% Cl 1.03-5) and tobacco abuse (OR 2.35, 95\% Cl 1.04-5.25) were independently associated with the development of any vasculopathy, whereas CART use was also an independent risk factor for the stenosis/occlusion subtype specifically (OR 2.87, 95\% Cl 1.117.45). Conclusions: There was a high frequency of cerebral arterial disease in this neuroimaging cohort of HIV/AIDS patients. A history of CART use and a history of tobacco abuse were independent risk factors for vasculopathy, though these findings should be confirmed with large-scale prospective studies.

๑) 2016 S. Karger AG, Basel

\section{Introduction}

Within the past decade, there has been a substantial rise in the number of stroke hospitalizations for HIV-infected patients, even in the face of a declining rate of stroke hospitalizations overall [1]. And though there is conflicting data, recent studies suggest an increased

\section{KARGER}

E-Mail karger@karger.com

www.karger.com/ced
(C) 2016 S. Karger AG, Basel

$1015-9770 / 16 / 0414-0170 \$ 39.50 / 0$
Nancy J. Edwards, MD

University of Texas Health Science Center at Houston

6431 Fannin Street, Rm 7.154

Houston, TX 77030-1503 (USA)

E-Mail Nancy.J.Edwards@ uth.tmc.edu 
stroke risk in HIV-infected patients compared with demographically and behaviorally similar uninfected patients [2]. One potential cause of stroke in HIV-infected patients is vascular disease of the cerebral vessels (e.g., cerebral vasculopathy). The earliest studies of vasculopathy as a direct consequence of HIV infection were in various animal models. Approximately $20 \%$ of rhesus monkeys infected with simian immunodeficiency virus (SIV) developed an arteriopathy characterized by intimal hyperplasia and fibrosis [3]. In another model, $60 \%$ of HIV1 transgenic mice harbored vascular lesions - including lesions of the cerebral vessels - similar to those observed in the SIV model [4]. Regarding HIV-infected patients, the first reports of clinically evident cerebral vasculopathy were in the pediatric AIDS population. The intracranial vessels of these children - all with high viral loads and low CD4 counts - were described as tortuous and ectatic, often with aneurysmal dilatations $[5,6]$. Case reports of cerebral vasculopathy in HIV-infected adults were subsequently published $[7,8]$. Observational studies of stroke in HIV-infected patients again noted the presence of vasculopathy in a subset of patients, intra- and extracranial, including both stenotic and aneurysmal lesions. These cohorts were relatively young and devoid of traditional risk factors for cardiovascular disease (CVD) $[9,10]$. The etiology of the arterial cerebrovascular disease in these HIV-infected patients was unclear, though proposed mechanisms included concomitant infectious vasculitides, HIV-induced endothelial dysfunction and even adverse effects of combination antiretroviral therapy (cART).

In this study, we sought to radiographically characterize and define the prevalence of cerebral arterial disease in a large neuroimaging cohort of HIV-infected adults in San Francisco. We hypothesized a relatively high frequency of vasculopathy in our HIV cohort and evaluated multiple HIV - and cART-related factors along with traditional CVD risk factors to try and identify the predictors of vasculopathy.

\section{Methods}

\section{Study Population}

We performed a search via The Health Record Data Service to identify all patients with an International Classification of Diseases, Ninth revision, code of 042 through 044 (HIV disease), V08 (asymptomatic HIV infection status) or 795.71 (non-specific serologic evidence of HIV) in the electronic database of patients treated at the San Francisco General Hospital during 2000-2013. HIV infection was confirmed by review of diagnostic laboratories
(HIV-1/HIV-2 antibody testing and/or detection of HIV-1 nucleic acids by polymerase chain reaction). A total of 6,298 HIV-infected patients were identified. We then screened the radiologic records of all HIV-infected patients to delineate our cohort of interest, which included those with cerebrovascular imaging of the intra- and extracranial vasculature via CT angiography, MR angiography or conventional angiography. One hundred forty-four HIV-infected patients with cerebrovascular imaging were identified and entered into our study.

The University of California at San Francisco Committee on Human Research approved this study.

\section{Data Extraction}

Detailed reviews of electronic medical records were performed for each patient. We extracted information regarding the extent and duration of HIV disease, comorbid cardiovascular conditions and CVD risk factors, inpatient and outpatient pharmacy history and relevant laboratories. cART regimens and duration were cross-checked with pharmacy records, and cocaine and/or methamphetamine use was determined by self-report in conjunction with urine toxicology reports (when available). The radiologic reports of all cerebrovascular imaging studies were reviewed, and the actual images were also interpreted retrospectively by an experienced neurologist (N.J.E.). We categorized the cerebrovascular findings into 3 groups: (1) normal vasculature, (2) dolichoectasia and/or intra- or extracranial aneurysms (dolichoectasia/aneurysm) and (3) flow-limiting vascular narrowing or occlusion (stenosis/occlusion).

\section{Statistical Analysis}

For the univariate analysis, the 2 vasculopathy subtypes (dolichoectasia/aneurysm and stenosis/occlusion) were individually assessed against the absence of vasculopathy. The Student's t test was used for continuous variables, and the Mann-Whitney U test and chi-square test were used for categorical variables. We then performed a multivariate analysis to identify those factors independently contributing to any vasculopathy, and a multinomial logistic regression to identify potential risk factors for the 2 vasculopathy subtypes compared to the absence of vasculopathy. The following variables were considered as candidates for inclusion in our multivariate/multinomial models: demographics, traditional CVD risk factors, HIV-related factors and all variables with $\mathrm{p}$ values $<0.02$ on univariate analysis. All analyses were conducted using SPSS version 21.0. Statistical significance was set at values of $\mathrm{p}<$ 0.05 .

\section{Results}

\section{Prevalence of Cerebral Arterial Disease in Our Study Cohort}

Of the 6,298 HIV-infected patients screened, $144 \mathrm{had}$ undergone cerebrovascular imaging of the intra- and extracranial vessels with CT, MR or conventional angiography. The presence of a focal neurologic deficit (67 patients) was the primary indication for imaging in this cohort, though altered mental status (30 patients), trauma 
Table 1. Baseline characteristics of HIV-infected patients with and without vasculopathy

\begin{tabular}{|c|c|c|c|c|c|}
\hline Gender, M:F & $71: 13$ & $12: 8$ & $0.01^{*}$ & $27: 8$ & 0.34 \\
\hline Nadir CD4, T cells/ $\mu \mathrm{l}$ & 237 & 229 & 0.90 & 216 & 0.63 \\
\hline cART (ever) & $42(50)$ & $12(60)$ & 0.42 & $26(74)$ & $0.02 *$ \\
\hline Duration of cART, years & 6.5 & 5.5 & 0.46 & 6.9 & 0.75 \\
\hline Protease inhibitor (ever) & $28(33)$ & $8(40)$ & 1 & $15(43)$ & 0.38 \\
\hline cART CPE & 7.3 & 6.7 & 0.46 & 7.5 & 0.63 \\
\hline Acute stroke identified at the time of vascular imaging & $59(70)$ & $13(65)$ & 0.85 & $19(54)$ & 0.15 \\
\hline Cocaine and/or methamphetamines (ever) & $51(61)$ & $13(72)$ & 0.72 & $18(51)$ & 0.35 \\
\hline Hyperlipidemia & $16(19)$ & $4(20)$ & 1 & $13(37)$ & $0.04^{*}$ \\
\hline Tobacco (ever) & $42(50)$ & $13(72)$ & 0.23 & $21(60)$ & 0.32 \\
\hline History of syphilis & $8(10)$ & $1(5)$ & 1 & $5(14)$ & 0.52 \\
\hline Clinical history of zoster & $9(11)$ & $3(15)$ & 0.70 & $4(11)$ & 1 \\
\hline Intracranial infection (ever) & $16(19)$ & $3(15)$ & 1 & $6(17)$ & 0.81 \\
\hline Intracranial neoplasm (ever) & $3(4)$ & $1(5)$ & 0.58 & $0(0)$ & 0.55 \\
\hline
\end{tabular}

Continuous variables are presented as means or $\mathrm{n}(\%)$. CPE $=$ CNS penetration effectiveness. ${ }^{*} \mathrm{p}<0.05$.

(16 patients), syncope (12 patients) and seizure (9 patients) were also frequent reasons.

Eighty-four of 144 patients (58.3\%) had normal intraand extracranial vascular imaging. Twenty (13.9\%) had a vasculopathy characterized by dolichoectasia - elongated, tortuous vessels - with or without aneurysms. Within this vasculopathy subtype, 10 patients had intracranial aneurysms without additional vessel findings, 9 patients had intracranial aneurysms in conjunction with vessel dolichoectasia, and 1 patient had multi-vessel dolichoectasia without discrete aneurysm formation. Three patients had multiple aneurysms. All the aneurysms were intracranial, with $76.2 \%$ located in the anterior circulation.

The other vasculopathy subtype we identified was essentially the polar opposite of dolichoectasia. This subtype - stenosis/occlusion - was characterized by flowlimiting vessel narrowing or frank vessel occlusion. Within our imaging cohort, 35 patients $(24.3 \%)$ had evidence of intra- and/or extracranial stenosis/occlusion. Twenty of these 35 patients had exclusively intracranial disease, whereas 11 had exclusively extracranial disease; 4 patients had diffuse disease involving both intra- and extracranial vessels. Of those with extracranial vessel involvement, the carotid artery was the stenosed or occluded vessel in 13 $(86.7 \%)$ patients, with moderate-to-severe carotid stenosis in 11 of these $13(84.6 \%)$.

Five patients $(3.5 \%)$ had 'other' vascular lesions - 3 patients had arteriovenous malformations and 2 patients had extracranial dissections that were likely traumatic in origin.

\section{Association of Vasculopathy with HIV-Related and Cerebrovascular Risk Factors}

The baseline characteristics of our HIV-infected cohort, as stratified by their cerebrovascular imaging findings, are outlined in table 1. Regarding traditional CVD risk factors, a history of diabetes and hyperlipidemia were relatively infrequent, whereas tobacco abuse and cocaine and/or methamphetamine use (both prior and active use) were fairly prevalent in our study cohort. Univariate analysis identified several variables associated with vasculopathy. Female gender and higher peak viral load were associated with the dolichoectasia/aneurysm subtype, whereas a history of cART use, hypertension and hyperlipidemia was associated with the stenosis/occlusion sub- 
Table 2. Multivariable analysis of factors contributing to any vasculopathy

\begin{tabular}{lll}
\hline Variable & $\begin{array}{l}\text { Adjusted } \\
\text { OR }(95 \% \mathrm{CI})\end{array}$ & p value \\
\hline Age & $1.09(0.67-1.76)$ & 0.74 \\
Gender & $2.49(0.99-6.3)$ & 0.05 \\
cART (ever) & $2.27(1.03-5)$ & $0.04^{*}$ \\
Peak viral load & $1.11(0.91-1.34)$ & 0.28 \\
Nadir CD4 count & $0.96(0.81-1.12)$ & 0.59 \\
Cocaine and/or methamphetamines (ever) & $0.76(0.33-1.8)$ & 0.52 \\
Hypertension & $1.40(0.6-3.27)$ & 0.43 \\
Diabetes & $1.55(0.44-5.43)$ & 0.49 \\
Hyperlipidemia & $1.29(0.51-3.24)$ & 0.59 \\
Tobacco (ever) & $2.35(1.04-5.25)$ & $0.04^{*}$ \\
\hline \multirow{2}{*}{$*$ p $<0.05}$. & & \\
\hline
\end{tabular}

Table 3. Multinomial regression analysis of factors contributing to the 2 vasculopathy subtypes

\begin{tabular}{|c|c|c|c|c|}
\hline Variable & $\begin{array}{l}\text { adjusted OR } \\
(95 \% \mathrm{CI})\end{array}$ & $\mathrm{p}$ value & $\begin{array}{l}\text { adjusted OR } \\
(95 \% \mathrm{CI})\end{array}$ & $\mathrm{p}$ value \\
\hline Age & $1.01(0.49-2.05)$ & 0.99 & $1.01(0.61-1.85)$ & 0.82 \\
\hline Gender & $3.09(0.92-10)$ & 0.07 & $2.1(0.7-6.33)$ & 0.19 \\
\hline cART (ever) & $2.02(0.64-6.37)$ & 0.23 & $2.87(1.11-7.45)$ & $0.03^{*}$ \\
\hline Cocaine and/or methamphetamines (ever) & $0.85(0.26-2.83)$ & 0.8 & $0.75(0.28-1.98)$ & 0.56 \\
\hline Hypertension & $1.58(0.48-5.15)$ & 0.45 & $1.33(0.50-3.57)$ & 0.58 \\
\hline Diabetes & $1.15(0.17-7.81)$ & 0.89 & $1.7(0.42-6.84)$ & 0.45 \\
\hline Hyperlipidemia & $0.88(0.22-3.5)$ & 0.85 & $1.7(0.6-4.8)$ & 0.59 \\
\hline Tobacco (ever) & $2.63(0.81-8.55)$ & 0.11 & $2.14(0.84-5.40)$ & 0.11 \\
\hline
\end{tabular}

$* \mathrm{p}<0.05$.

type. The mean peak viral load in patients with normal vessels was 126,114 copies $/ \mathrm{ml}$ in contrast to $317,161 \mathrm{cop}$ ies $/ \mathrm{ml}$ in those with vessel dolichoectasia and/or aneurysms.

Multivariate analysis was then performed to delineate (1) those variables independently associated with any vasculopathy (as compared to patients without vasculopathy) and (2) those variables predictive of vasculopathy subtype specifically (dolichoectasia/aneurysm vs. stenosis/occlusion). The results are provided in tables 2 and 3. Following adjustment for demographics, HIV-related factors and traditional CVD risk factors, a history of cART use (adjusted OR 2.27, 95\% CI 1.03-5) along with a history of tobacco abuse (adjusted OR 2.35, 95\% CI 1.04-5.25) were independently associated with the devel- opment of any vasculopathy. Of those patients with vasculopathy, a history of cART use (adjusted OR 2.87, 95\% CI 1.11-7.45) was independently associated with the stenosis/occlusion subtype. No variables were independently associated with the dolichoectasia/aneurysm subtype, though female gender approached significance (adjusted OR 3.09, 95\% CI 0.92-10).

\section{Discussion}

Our study is the largest study to date of radiographically evident cerebral vasculopathy in HIV-infected patients. The frequency of cerebral arterial disease in our study cohort was high: 55 patients, $38.2 \%$, had vascular 
abnormalities on neurovascular imaging obtained for a variety of indications. These vascular abnormalities were divided into 2 subtypes given probable contrasting pathophysiologies: (1) dolichoectasia/aneurysm, likely to be characterized pathologically by atrophy of the media and fragmentation of the elastic lamina, and (2) stenosis/occlusion, likely to be secondary to intimal hyperplasia and smooth muscle cell proliferation, akin to atherosclerosis. We discovered the prevalence of both vasculopathy subtypes to be quite high in our cohort, as $13.9 \%$ of patients had dolichoectatic/aneurysmal vessels and $24.3 \%$ had stenotic or occluded vessels. This is in sharp contrast to historical prevalence studies of the general population. One systematic review estimated the prevalence of cerebral aneurysms in the general population to be $2 \%$ [11]. Even in populations enriched for aneurysm risk factors - such as acute ischemic stroke patients - the prevalence of cerebral aneurysms has been reported as only $6.6-9.3 \%[12,13]$. Regarding those patients with stenotic/occluded vessels, 11 patients, $7.6 \%$ of our cohort, had evidence of moderate-to-severe carotid stenosis, the mean age of this subset being 51.9 years. Contrary to this, previously published studies of the general population estimate a $0.5-1 \%$ prevalence of moderate-to-severe carotid stenosis in patients under the age of 60 years [14].

The high frequency of vasculopathy reported here should be interpreted with several study limitations in mind. First, our study cohort is a cohort of HIV-infected patients referred for neuroimaging; as a result, there may be a degree of selection bias. Nevertheless, the indications for imaging were varied; in fact, $11 \%$ of patients received imaging simply due to a history of head and neck trauma. Another caveat to the interpretation of our study is the single site nature, particularly as high rates of drug abuse (cocaine/methamphetamines, tobacco) were noted in our study subjects. Cocaine- and methamphetamine-induced vasculopathy have been previously described, and in one study of stroke in cocaine users, 12 of 45 patients were noted to have carotid stenosis during their stroke evaluation [15]. In the stroke study, though, patients had high rates of concomitant CVD risk factors whereas our study subjects had considerably lower rates of diabetes and hyperlipidemia (and to a lesser degree, hypertension). Furthermore, active and/or prior cocaine/methamphetamine use was prevalent across all our study groups (e.g., patients with and without vasculopathy); only tobacco abuse was independently associated with vasculopathy in our cohort. And lastly, one could argue that the prevalence of drug abuse in our cohort reinforces the generalizability of our study results to the greater HIV population. Similar rates of drug abuse were noted in the Fat Redistribution and Metabolic Change in HIV Infection cohort with 16 participating clinical sites across the United States, and studies of HIV-infected patients in Sub-Saharan Africa have also documented equivalently high drug abuse rates $[10,16]$.

The pathogenesis of cerebral arteriopathy in HIV is likely multifactorial. Hypotheses regarding pathophysiology range from vasculitis due to non-HIV infectious agents, direct viral invasion of vascular cells and/or endothelial injury mediated by HIV proteins, chronic immune dysregulation and inflammation and toxicities of HIV drug regimens $[9,17-20]$. Several patients in our cohort likely had an infectious cerebral vasculitis; 1 patient with vessel dolichoectasia and multiple intracranial aneurysms had biopsy-proven varicella zoster virus vasculitis. Though our study was not one of vasculitis per se, we did not find a significant correlation of cerebral arteriopathy with a history of syphilis, clinical history of zoster, hepatitis B and/or C co-infection or a history of intracranial infection. This should be interpreted with caution, though - as our study was retrospective, we do not have comprehensive infectious serologies and/or cerebrospinal fluid results for all our patients.

Although HIV is unlikely to be vasculotropic, vascular cells are continually exposed to HIV-infected leukocytes, viral proteins and viral-induced proinflammatory cytokines [21]. Plasma concentrations of multiple inflammatory biomarkers are significantly elevated in HIV-infected patients compared with controls, and several of these biomarkers have been linked to endothelial injury and atherosclerosis [22-24]. For instance, in one study of carotid intima-media thickness (IMT) in HIV-infected patients, higher levels of high sensitivity C-reactive protein (hsCRP) correlated with both higher levels of IMT and faster progression of IMT in the carotid bifurcation region [25]. In the prospective SUN study, HIV-infected patients with suppressed viral loads throughout the study period had significantly less CIMT progression as compared to patients with detectable viral loads [26]. Similarly, we sought to determine whether HIV-related factors such as peak viral load, nadir CD4 count and duration of HIV correlated with the presence of cerebral vasculopathy in our study subjects. In the univariate analysis, higher peak viral loads were associated with the dolichoectasia/aneurysm vasculopathy subtype, though this was not reproduced in the multivariate analysis. This may in part be due to the relatively small number of patients in the dolichoectasia/aneurysm subtype (20 patients vs. 
84 with normal vessels), and therefore warrants further prospective evaluation. In one relatively large study of intracranial arterial samples obtained at autopsy, HIV-infected patients with protracted infection and/or detectable viral loads at death had significantly higher rates of dolichoectasia [27].

Interestingly, in our study subjects, the use of cART was significantly and independently associated with cerebral vasculopathy even when controlling for HIV disease severity, with the use of cART conferring a nearly 3 -fold increase in the risk of cerebrovascular disease. Furthermore, cART was predictive for the stenosis/occlusion vasculopathy subtype - the subtype whose pathophysiology is likely akin to atherosclerosis. Though controversy exists, several lines of evidence suggest long-term use of cART may contribute to vascular disease, particularly accelerated atherosclerosis $[17,28-30]$. Certain antiretroviral drugs (protease inhibitors) may result in metabolic disorders such as insulin resistance and dyslipidemia. Several studies suggest abacavir, a nucleotide reverse transcriptase inhibitor, may upregulate various proinflammatory cytokines (hsCRP, interleukin-6) in patients with HIV [31]. As HIV-infected individuals receiving cART are likely to live longer, they may do so with longterm metabolic challenges and/or the ramifications of chronic systemic inflammation, potentially increasing vascular disease risk. Therefore, vigilance in minimizing additional vascular risk factors in patients receiving cART is likely advisable. In our study subjects, tobacco abuse was the other variable independently associated with vasculopathy; it has yet to be seen if tobacco abuse and cART have additive effects upon cerebrovascular disease and/or events.

\section{Conclusion}

In conclusion, in this neuroimaging cohort of HIVinfected patients, the frequency of cerebral arterial disease was high. Two types of vasculopathy were identified - dolichoectasia with or without intracranial aneurysms, and stenosis or frank occlusion of intra- or extracranial vessels. The use of cART and abuse of tobacco were independently associated with any vasculopathy, with the use of CART also being an independent predictor of the stenosis/occlusion vasculopathy subtype. Although higher peak viral loads and female gender were also identified in the univariate analysis as predictive of the dolichoectasia/aneurysm subtype, this was not confirmed in the multivariate analysis. Further prospective epidemiological studies are warranted, as are studies exploring the pathophysiology, progression and the clinical ramifications of cerebrovascular disease in HIV-infected patients (e.g., stroke, HIV-associated neurocognitive disorders).

\section{Disclosure Statement}

The authors have no conflicts of interest to declare.

\section{Study Funding}

This publication was supported by the National Center for Research Resources and the National Center for Advancing Translational Sciences, National Institutes of Health, through UCSF-CTSI grant number UL1 RR024131. Its contents are solely the responsibility of the authors and do not necessarily represent the official views of the NIH.

\section{References}

1 Ovbiagele B, Nath A: Increasing incidence of ischemic stroke in patients with HIV infection. Neurology 2011;76:444-450.

2 Sico JJ, Chang CC, So-Armah K, Justice AC, Hylek E, Skanderson M, et al: HIV status and the risk of ischemic stroke among men. Neurology 2015;84:1933-1940.

3 Chalifoux LV, Simon MA, Pauley DR, MacKey JJ, Wyand MS, Ringler DJ: Arteriopathy in macaques infected with simian immunodeficiency virus. Lab Invest 1992;67:338-349.

4 Tinkle BT, Ngo L, Luciw PA, Maciag T, Jay G: Human immunodeficiency virus-associated vasculopathy in transgenic mice. J Virol 1997; 71:4809-4814.

5 Dubrovsky T, Curless R, Scott G, Chaneles M, Post MJ, Altman N, et al: Cerebral aneurysmal arteriopathy in childhood AIDS. Neurology 1998;51:560-565.

6 Shah SS, Zimmerman RA, Rorke LB, Vezina LG: Cerebrovascular complications of HIV in children. AJNR Am J Neuroradiol 1996;17: 1913-1917.

7 Stanley A, Candy S, Levin C, Heckmann JM: The complexity of HIV vasculopathy. S Afr Med J 2012;102:474-476.

8 Kossorotoff M, Touzé E, Godon-Hardy S, Serre I, Mateus C, Mas JL, et al: Cerebral vasculopathy with aneurysm formation in HIV-infected young adults. Neurology 2006;66:1121-1122.

9 Ortiz G, Koch S, Romano JG, Forteza AM, Rabinstein AA: Mechanisms of ischemic stroke in HIV-infected patients. Neurology 2007;68:1257-1261.
10 Tipping B, de Villiers L, Wainwright H, Candy S, Bryer A: Stroke in patients with human immunodeficiency virus infection. J Neurol Neurosurg Psychiatry 2007;78:1320-1324.

11 Rinkel GJ, Djibuti M, Algra A, van Gijn J: Prevalence and risk of rupture of intracranial aneurysms: a systematic review. Stroke 1998; 29:251-256.

12 Oh YS, Lee SJ, Shon YM, Yang DW, Kim BS, Cho AH: Incidental unruptured intracranial aneurysms in patients with acute ischemic stroke. Cerebrovasc Dis 2008;26:650-653.

13 Edwards NJ, Kamel H, Josephson SA: The safety of intravenous thrombolysis for ischemic stroke in patients with pre-existing cerebral aneurysms: a case series and review of the literature. Stroke 2012;43:412-416. 
14 Whitty CJ, Sudlow CL, Warlow CP: Investigating individual subjects and screening populations for asymptomatic carotid stenosis can be harmful. J Neurol Neurosurg Psychiatry 1998;64:619-623.

15 Toossi S, Hess CP, Hills NK, Josephson SA: Neurovascular complications of cocaine use at a tertiary stroke center. J Stroke Cerebrovasc Dis 2010;19:273-278.

16 Cofrancesco J Jr, Scherzer R, Tien PC, Gibert CL, Southwell H, Sidney S, et al: Illicit drug use and HIV treatment outcomes in a US cohort. AIDS 2008;22:357-365.

17 Benjamin LA, Bryer A, Emsley HC, Khoo S, Solomon T, Connor MD: HIV infection and stroke: current perspectives and future directions. Lancet Neurol 2012;11:878-890.

18 Goldstein DA, Timpone J, Cupps TR: HIVassociated intracranial aneurysmal vasculopathy in adults. J Rheumatol 2010;37:226-233.

19 Nagel MA: Varicella zoster virus vasculopathy: clinical features and pathogenesis. J Neurovirol 2014;20:157-163.

20 Sen S, Rabinstein AA, Elkind MS, Powers WJ: Recent developments regarding human immunodeficiency virus infection and stroke. Cerebrovasc Dis 2012;33:209-218.
21 Baliga RS, Chaves AA, Jing L, Ayers LW, Bauer JA: AIDS-related vasculopathy: evidence for oxidative and inflammatory pathways in murine and human AIDS. Am J Physiol Heart Circ Physiol 2005;289:H1373-H1380.

22 Hunt PW: HIV and inflammation: mechanisms and consequences. Curr HIV/AIDS Rep 2012;9:139-147.

23 Neuhaus J, Jacobs DR Jr, Baker JV, Calmy A, Duprez D, La Rosa A, et al: Markers of inflammation, coagulation, and renal function are elevated in adults with HIV infection. J Infect Dis 2010;201:1788-1795.

24 Parikh RV, Scherzer R, Nitta EM, Leone A, Hur S, Mistry V, et al: Increased levels of asymmetric dimethylarginine are associated with pulmonary arterial hypertension in HIV infection. AIDS 2014;28:511-519.

25 Hsue PY, Scherzer R, Hunt PW, Schnell A, Bolger AF, Kalapus SC, et al: Carotid intimamedia thickness progression in $\mathrm{HIV}$-infected adults occurs preferentially at the carotid bifurcation and is predicted by inflammation. J Am Heart Assoc 2012;pii:jah3-e000422.
26 Baker JV, Henry WK, Patel P, Bush TJ, Conley LJ, Mack WJ, et al: Progression of carotid intima-media thickness in a contemporary human immunodeficiency virus cohort. Clin Infect Dis 2011;53:826-835.

27 Gutierrez J, Goldman J, Dwork AJ, Elkind MS, Marshall RS, Morgello S: Brain arterial remodeling contribution to nonembolic brain infarcts in patients with HIV. Neurology 2015;85:1139-1145.

28 Di Biagio A, Del Bono V, Rosso R, Viscoli C: HIV and accelerated atheroprogression: role of antiretroviral therapy. Curr Pharm Biotechnol 2012;13:88-96.

29 Friis-Moller N, Sabin CA, Weber R, d'Arminio Monforte A, El-Sadr WM, Reiss P, et al: Combination antiretroviral therapy and the risk of myocardial infarction. N Engl J Med 2003; 349:1993-2003.

30 Singer EJ, Valdes-Sueiras M, Commins DL, Yong W, Carlson M: HIV stroke risk: evidence and implications. Ther Adv Chronic Dis 2013;4:61-70.

31 Kuller LH, Tracy R, Belloso W, De Wit S, Drummond F, Lane HC, et al: Inflammatory and coagulation biomarkers and mortality in patients with HIV infection. PLoS Med 2008; 5:e203. 